\title{
Uso del Test de Rendimiento Continuo de Conners para diferenciar niños normales y con TDAH en Chile.
}

\section{Using the Conners Continous Performance Test for differentiation of normal and ADHD Chilean children.}

\author{
Sonia Salas-Bravo \\ Universidad de La Serena, Centro de Estudios Avanzados en Zonas Áridas (CEAZA), Chile. \\ Mauricio Gonzalez-Arias \\ Departamento de Psicología. Universidad de La Serena, Chile. \\ Angelo Araya-Piñones \\ Universidad de La Serena, Chile. \\ Macarena Valencia-Jimenez \\ Ilustre Municipalidad de Salamanca, Chile. \\ Sonia Oyarce-Cortes C. \\ Corporación de Oportunidad y Acción Solidaria (OPCIÓN), Chile.
}

Recepción (11 de febrero de 2015) Aceptado (03 de noviembre de 2017)

\begin{abstract}
Resumen
El trastorno de déficit atencional con hiperactividad (TDAH) constituye uno de los cuadros de mayor prevalencia durante la niñez. El presente trabajo se focalizó en evaluar si el Test de Rendimiento Continuo de Conners era capaz de discriminar entre niños con y sin TDAH diagnosticados por el docente. Se conformó una muestra no probabilística de 30 niños escolares (15 clínicos y 15 normales) a través de la aplicación del cuestionario de atención. Todos los niños completaron la aplicación del test computarizado de Conners. Se observaron diferencias significativas entre la muestra normal y clínica. Todos los niños seleccionados como normales no encajaron el perfil clínico. Solo el 50\% de los casos considerados con TDAH encajaron el perfil clínico. Se analizan las implicancias del sobre/diagnóstico del trastorno.

Palabras Clave: TDAH, test de Rendimiento Continuo, diagnóstico, escalas diagnósticas.
\end{abstract}

\begin{abstract}
Attention-deficit hyperactivity disorder (ADHD) is one of the most prevalent disorder during childhood. The present study aimed to evaluate if the Conners Continous Performance Test was able to discriminate among $\mathrm{ADHD}$ and normal children completed the Conners computarized test. Significant differences between normal and clinical sample were found. All children selected as normal did not fit the clinical profile. Only $50 \%$ of the children considered as having ADHD fit the clinical profile. Implications for the issue of overdiagnosis of the disorder are discussed.

Key Words: ADHD; Continous Performance Test; diagnosis; diagnosis scales.
\end{abstract}

Correspondencia: Universidad de La Serena/CEAZA, Benavente 980, La Serena., salassc@yahoo.com, mgonzale@userena.cl, angelo.arayap@gmail. com,makyroni@gmail.com, sonia.oyarce.cortes@gmail.com 


\section{Introducción}

El trastorno por déficit atencional con hiperactividad (TDAH) también denominado trastorno hipercinético con hiperactividad, constituye una de las alteraciones psicopatológicas más estudiadas actualmente en el área de la salud mental infantil. La sintomatología básica del trastorno se caracteriza por alteraciones de la atención, pudiendo acompañarse de hiperactividad y/o impulsividad, observándose efectos importantes en el funcionamiento académico, interpersonal y/o social (Antshel et al. 2011). Los síntomas del trastorno estarían parcialmente vinculados con mecanismos neuropsicológicos y rasgos temperamentales con resultados diferenciales en la adolescencia y la adultez (Martell y Niggs, 2006).

La prevalencia global del trastorno en la población presenta variaciones considerables dependiendo del método de evaluación, las características del muestreo y la edad del niño (Sciutto y Eisenberg, 2007). Las tasas de prevalencia a nivel internacional fluctuarían entre 3\% y 7\% según Monastra (2008) y la American Psychiatric Association (2000); mencionándose también en la literatura un $8,7 \%$ (Wolraich et al. 2014). Las cifras aportadas por la reciente aparición del DSM-5 proporcionan un valor igual a 5\% (American Psychiatric Association, 2013).

La discrepancia en las tasas de prevalencia unido a la presencia de comorbilidad del trastorno, habrían despertado la inquietud entre los investigadores, quienes alzaron su voz en demanda de instrumentos de diagnóstico y evaluación que hicieran posible identificar en forma más precisa y objetiva el trastorno de TDAH. Algunas de las razones que incidirían en la variabilidad de las tasas de prevalencia del trastorno se relacionarian, de acuerdo a Berger (2011), con las definiciones y criterios diagnósticos diferenciales de las clasificaciones actualmente en uso; asimismo, se tornaría evidente el sesgo diagnóstico producto del juicio subjetivo de quienes deben evaluar el trastorno. Gupta y Kar (2010) señalan que la comorbilidad del trastorno provocaría superposición de los síntomas y con ello distorsión de los juicios evaluativos. Así, las dificultades del diagnóstico causarían discrepancias en la identificación y potencial sobre identificación del TDAH (Winders y Williams, 2011).

Son precisamente las condiciones de discrepancia en relación al diagnóstico, la fluctuación de las tasas de prevalencia y comorbilidad, unido a la tendencia descriptiva de los criterios de clasificación diagnóstica de mayor uso en las décadas precedentes (DSM-IV-R, CIE-10), las que habrían favorecido la emergencia de una serie de instrumentos (cuestionarios y autoinformes), constituyéndose en mecanismos relevantes del proceso diagnóstico del trastorno (Forbes, 2001). La revisión de las escalas de uso frecuente permite advertir que algunos autores (Brown, 2001) han desarrollado instrumentos considerando la evaluación más específica de funciones a través de sub-escalas (BADDS), o bien permitiendo medir en forma paralela (Escala de TDAH de Vanderbilt) la comorbilidad del trastorno (Wolraich et al. 2003).

En las últimas décadas se ha observado un creciente interés en desarrollar y utilizar instrumentos de medición computarizados (comúnmente denominadas CPT) cuya administración y resultados de carácter más objetivo, pudiesen enriquecer el proceso diagnóstico del TDAH. Entre las pruebas de rendimiento continuo (CPT) de uso frecuente, se destacan algunas como: el Gordon Diagnostic System (GDS) (Gordon 1983); el Test of Variables of Attention (TOVA) (Greenberg y Waldman, 1993); el Conners' Continuous Performance Task, -2, y -3 (CCPT) (Conners, 1995, 2000 y 2014), el Intermediate Visual and Auditory Continuous Performance Test (IVA) (Sandford, 1995) y el MOXO-CPT (Berger, Slobodin, Aboud, Melamed y Cassuto, 2013). Existen también instrumentos donde se utiliza la realidad virtual; tal es el caso del Virtual Reality-Continuous Performance Tasks (VRCPT) (Rizzo et al. 2006) y el AULA NESPLORA (Díaz-Orueta et al. 2014). Un atributo común de la mayoría de los CPT implica la exposición secuencial a estímulos presentados computacionalmente (figuras, números o letras), durante un espacio de tiempo predeterminado (Cohen, 2011). La mayoría de estos instrumentos mide el número de estímulos identificados correctamente, así como el tiempo de reacción (inter e intra serie de estímulos). Entre las ventajas de este tipo de pruebas se destaca el que son económicas, de fácil administración, libres de sesgo, pudiendo administrarse en ambientes diferentes (Nichols y Waschbusch, 2004); asimismo, su estructura facilitaría las labores de investigación comparada (Miranda et al., 2012).

La efectividad de las pruebas computarizadas ha sido analizada a través de estudios comparativos incluyendo variables asociadas al proceso de enseñanza. La revisión bibliográfica sin embargo, tendería a presentar resultados inconsistentes. Así, se observarían correlaciones significativas entre variables sintomáticas del TDAH según escalas para profesores y pruebas computarizadas (Halperin et al. 1988; Klee y Garfinkel, 1983 y Shapiro y Garfinkel, 1986). Por otra parte se destacan relaciones inconsistentes, bajas o ausentes al comparar diversas pruebas computarizadas y escalas administradas a docentes (Alloway et al. 2009; Barkley, 1991; 
DuPaul, Anastopoulos, Shelton, Guevremont, y Metevia, 1992; Kallitsoglou, 2013; McGee, Clark y Symons, 2000; Muir-Broaddus, Rosenstein, Medina y Soderberg, 2002 y Weiss y Totten, 2004). Resultados similares a los señalados anteriormente se evidenciarían cuando se comparan los instrumentos computarizados con resultados de escalas aplicadas conjuntamente a padres y profesores (Edwards et al. 2007; Egeland, Johansen y Ueland, 2009; Naglieri, Goldstein, Delauder y Schwebach, 2005 y Nigg, Hinshaw y Halperin, 1996)

En términos del rol ejercido por los distintos actores del sistema hogar-escuela, se evidencia que si bien los docentes no habrían recibido una capacitación sistemática sobre el tema (Bell, Long, Garvan y Bussing, 2011), poseerían competencias para la identificación del TDAH (Anderson, Watt, Noble y Shanley, 2012), identificando subtipos (Moldavsky, Groenewald, Owen y Sayal, 2013), realizando esta labor mejor incluso que los propios padres (Tripp, Schaughency y Clarke, 2006); esta condición permitiría considerar al cuerpo docente como intérpretes y conocedores de los indicadores del TDAH.

En síntesis, la revisión anterior si bien considera importante el aporte de los docentes en el proceso de apoyo y tratamiento de los niños que presentan TDAH, denota discrepancias en torno a la correspondencia de diagnóstico del trastorno, entre los instrumentos de evaluación y el juicio evaluativo de los profesores. Este hecho apunta a la necesidad de realizar trabajos que midan la capacidad discriminativa de las pruebas utilizadas en la actualidad, contrastándolas con el diagnóstico realizado por los académicos encargados de los procesos docentes.

En consideración al planteamiento anterior, el presente trabajo tuvo como objetivo evaluar la capacidad discriminativa de uno de los test computarizados de uso frecuente hoy en día (CPT-II) diferenciando entre niños considerados normales y niños con trastorno de déficit atencional con hiperactividad, según el criterio proporcionado por profesores a través de una escala de atención y adaptación.

\section{Método}

\section{Participantes}

Se conformó una muestra no probabilística intencionada compuesta por 30 niños, cuyas edades fluctuaron entre 8 y 13 años, con una edad promedio de 10.9 años $(D E=2.89)$. Un $63.3 \%$ de la muestra $(n=19)$ eran mujeres y un $36.7 \%(n=11)$ hombres, pertenecientes a niveles escolares entre segundo y sexto año de enseñanza básica. Los participantes provenían de dos colegios municipales de la Región de Coquimbo (Chile) de estrato socio económico medio-bajo. Se conformaron dos sub-muestras: 15 niños compusieron la muestra "clínica", es decir niños diagnosticados con TDAH de acuerdo al criterio de los docentes; 15 niños configuraron la muestra "no clínica" siguiendo el mismo criterio de selección. Las dos sub muestras poseían características similares en relación a la edad, sexo y estrato socio-económico.

\section{Instrumentos}

Children's Attention and Adjusment Survey School Form (CAAS-S) (Lambert, Hartsough y Sandoval, 1990): La escala es de tipo Likert ( 0 a 3 puntos) y consta de 31 items. Está diseñada para medir los criterios diagnósticos del TDAH e incluye cuatro sub-escalas: desatención, impulsividad, hiperactividad y problemas de conducta-agresividad. Se utilizó la forma escolar que está diseñada para ser respondida por el docente y es aplicable a niños entre 5 y 13 años. Se corrige en forma manual obteniéndose un gráfico con el perfil expresado en percentiles. De acuerdo a los autores, el grado de confiabilidad (alpha) osciló entre .78 y .94 en tanto la validez se consideró aceptable. Para los fines del presente trabajo la versión en inglés del instrumento se tradujo y adaptó al español siguiendo todos los procedimientos estándares para estos fines y ha conservado la consistencia interna en la muestra estudiada.

Conners Continous Performance Test II (CPT-II) (Conners, 2004): Prueba computarizada que mide atención sostenida; está compuesta por estímulos visuales proyectados en la pantalla del computador, donde el sujeto debe presionar la barra espaciadora ante series de letras a excepción de un distractor (letra $\mathrm{X}$ ). Los estímulos visuales se presentan en seis bloques con 20 letras dada uno, mediando intervalos de 1 , 2 y 4 segundos. La prueba tiene una duración de 14 minutos y está estandarizada para sujetos de 6 años en adelante. El manual del test informa niveles de confiabilidad que varían entre .66 y .95 a través del método de mitades fluctuantes (Conners, 2004); los valores de correlación usando el metodo test-retest variaron de .05 a .92 . El autor concluye que la prueba posee capacidad para diferenciar entre grupos clínicos y no clínicos. Homack y Riccio (2006) a su vez señalan que la prueba constituye una fuente objetiva de información, la cual se puede complementar con la información disponible al momento del diagnóstico. 


\section{Procedimiento}

Se realizaron reuniones informativas con los directores de los establecimientos, obteniéndose consentimiento informado firmado de los padres de los niños participantes. Se solicitó a los profesores que seleccionaran a aquellos alumnos que a su juicio cumplieran con los criterios diagnósticos del trastorno de déficit atencional con hiperactividad. Se administró a los docentes la escala CAAS para evaluar a los niños seleccionados; la muestra "clínica" que inicialmente fue de 22 niños, se redujo a 15. A continuación se solicitó a los mismos profesores que seleccionaran 15 niños que a su juicio fueran considerados "sin TDAH", aplicándoles la escala CAAS para asegurarse que no fueran diagnosticados con TDAH; se tomó precaución a fin que la submuestra "Sin TDAH" seleccionada cumpliera con las características etarias, de sexo y nivel socioeconómico de la sub muestra "clínica". A continuación se administró el test computarizado de rendimiento continuo (CPT-II) a la muestra final compuesta por 30 niños. El procesamiento de los datos se realizó mediante el software SPSS 11.5 empleándose la prueba exacta de Fisher.

\section{Resultados}

Se contrastaron las frecuencias de diagnósticos realizadas a partir del juicio del profesorado (CAAS-S), con el que se constituyeron los grupos, con la evaluación computarizada (CPT-II) aplicada posteriormente a los y las menores. La tabla de contingencia de $2 \mathrm{X} 2$ (ver Tabla 1) muestra que de la frecuencia inicial de 15 menores diagnosticados con presencia de TDAH (muestra clínica), solo 7 encajaron el perfil clínico de acuerdo al test computarizado. La diferencia de estas frecuencias fue estadísticamente significativa $\chi^{2}$ $(1, N=30)=9.13$, estadístico exacto de Fischer $p=.003$.

Tabla 1. Diagnóstico de TDAH en muestra clínica (CAAS-S) y según resultados de test computarizado (CPTII).

\begin{tabular}{l|llll|ll}
\hline \multirow{2}{*}{ Muestra } & \multicolumn{4}{|l|}{ Perfil clínico CPT-II } & \multirow{2}{*}{ Total } \\
\cline { 2 - 6 } & Se ajusta & No se ajusta & & \\
\hline & f & $\%$ & f & $\%$ & f & $\%$ \\
Con TDAH (CAAS-S) & 7 & $23 \%$ & 8 & $27 \%$ & 15 & $50 \%$ \\
Sin TDAH (CAAS-S) & 0 & $0 \%$ & 15 & $50 \%$ & 15 & $50 \%$ \\
Total & 7 & $23 \%$ & 23 & $77 \%$ & 30 & $100 \%$ \\
\hline
\end{tabular}

También, se analizaron las frecuencias de diagnósticos en relación al sexo de los participantes. En primer lugar se analizó la relación entre el sexo y el diagnóstico de TDAH según el perfil cínico obtenido por medio de la prueba computarizada (CPT) y los resultados no mostraron una relación significativa $\chi^{2}(1, N=30)=.258$, estadístico exacto de Fischer $p=.485$. La tabla 2, sintetiza el resultado de los análisis separados según el sexo y muestra que de los 10 niños que fueron diagnosticados con TDAH, con el test CAAS-S respondido por profesores solo 5 se ajustaron a los criterios del test CPT-II. A su vez, los 9 niños sin TDAH mantuvieron su diagnóstico negativo. La diferencia de estas frecuencias fue estadísticamente significativa $\chi^{2}(1, N=30)=6.12$, estadístico exacto de Fischer $p=.022$. En el caso de las niñas, de las 5 que conformaban la muestra con diagnóstico de TDAH, solo 2 lo mantuvieron con el CPT-II, pero este cambio de frecuencias no resultó significativo $\chi^{2}(1, N=30)=2.93$, estadístico exacto de Fischer $p=.182$ (ver Tabla 2).

Tabla 2. Diagnóstico de TDAH según sexo en muestra clínica (CAAS-S) y test computarizado (CPT-II)

\begin{tabular}{|c|c|c|c|c|c|c|c|}
\hline \multirow{3}{*}{ Sexo } & \multirow{3}{*}{$\begin{array}{l}\text { Muestra } \\
\text { CAAS-S }\end{array}$} & \multicolumn{4}{|c|}{ Perfil clínico CPT-II } & \multicolumn{2}{|c|}{ Total } \\
\hline & & \multicolumn{2}{|c|}{ se ajusta } & \multicolumn{2}{|c|}{$\begin{array}{l}\text { no se } \\
\text { ajusta }\end{array}$} & & \\
\hline & & $f$ & $\%$ & $\mathrm{f}$ & $\%$ & $\mathrm{f}$ & $\%$ \\
\hline \multirow[t]{3}{*}{ Masculino } & Con TDAH & 5 & $17 \%$ & 5 & $17 \%$ & 10 & $33 \%$ \\
\hline & Sin TDAH & 0 & $0 \%$ & 9 & $30 \%$ & 9 & $30 \%$ \\
\hline & Total & 5 & $17 \%$ & 14 & $47 \%$ & 19 & $63 \%$ \\
\hline \multirow[t]{3}{*}{ Femenino } & Con TDAH & 2 & $7 \%$ & 3 & $10 \%$ & 5 & $17 \%$ \\
\hline & Sin TDAH & 0 & $0 \%$ & 6 & $20 \%$ & 6 & $20 \%$ \\
\hline & Total & 2 & $7 \%$ & 9 & $30 \%$ & 11 & $37 \%$ \\
\hline Total & & 7 & $23 \%$ & 23 & $77 \%$ & 30 & $100 \%$ \\
\hline
\end{tabular}

Finalmente, en la Tabla 3 se reporta la relación entre la edad, expresada en 2 categorías (de 8 a 10 años una y de 11 a 16 años la otra) y el diagnóstico de TDAH por medio del CPT-II. Simultáneamente, en la misma tabla se reporta el nivel de escolaridad, también expresado en dos categorías (de $2^{\circ}$ a $4^{\circ}$ básico una, y de $5^{\circ}$ a $6^{\circ}$ básico otra) en relación al diagnóstico de TDAH por medio del CPT-II, ya que equivale exactamente a los mismos participantes. No se encontró relación significativa siendo $\chi^{2}(1, N=30)=.053$, estadístico exacto de Fischer $p=.182$ (ver Tabla 3 ). 
Macarena Valencia-Jimenez y Sonia Oyarce-Cortes. C

Tabla 3. Diagnóstico de TDAH con test computarizado (CPT-II) según edad o escolaridad

\begin{tabular}{ll|llll|ll}
\hline \multirow{2}{*}{ Variable } & Categoría & \multicolumn{2}{l|}{ Perfil clínico CPT-II } & \multicolumn{2}{l}{ Total } \\
& & \multicolumn{2}{l}{ Se ajusta } & No se ajusta & & \\
\hline \multirow{3}{*}{ Edad $/$} & 8 a 10 años $/ 2^{\circ}$ a $4^{\circ}$ básico & 3 & $10 \%$ & 11 & $37 \%$ & 14 & $47 \%$ \\
Escolaridad & 11 a 16 años $/ 5^{\circ}$ a $6^{\circ}$ básico & 4 & $13 \%$ & 12 & $40 \%$ & 16 & $53 \%$ \\
& Total & 7 & $23 \%$ & 23 & $77 \%$ & 30 & $100 \%$ \\
\hline
\end{tabular}

\section{Discusión y Conclusiones}

El presente trabajo tuvo como propósito determinar si la prueba de Rendimiento Continuo de Conners (CPT) era capaz de identificar niños con y sin TDAH en relación con el diagnóstico obtenido a partir del criterio de los profesores expresado por medio de la prueba CAAS-S. Los hallazgos del estudio en un nivel general apuntan a que la prueba computarizada de Conners presenta adecuados niveles de detección y una apropiada capacidad discriminativa entre muestras clínicas y no clínicas, lo cual ratifica lo reportado en los estudios del validación del instrumento (Conners, 2004). Por otra parte, no existiría una concordancia expresa y amplia entre el criterio del profesor, obtenido mediante el CAAS-S y los perfiles entregado por CPT de Conners. Presentándose en el caso de los profesores una mayor cantidad de falsos positivos respecto al CPT, lo que podría ser un indicador de sobre diagnóstico del TDAH en niños escolares, cuando la detección depende del docente. Determinar si hay o no un sobre diagnóstico dependerá en última instancia de un mayor consenso sobre cuáles deben ser los criterios críticos para realizar el diagnóstico y la pertinencia de la utilización de indicadores a partir de apreciaciones subjetivas o del rendimiento del niño en pruebas objetivas.

Respecto a la concordancia entre las mediciones, los resultados obtenidos por este estudio, son similares a los reportados el literatura especializada que han explorado la relación entre medidas computarizadas y escalas para profesores, sin encontrar una relación sistemática entre ambos tipos de medición (Alloway et al. 2009; Barkley, 1991; DuPaul et al. 1992; Edwards et al. 2007; Egeland et al. 2009; Kallitsoglou, 2013; McGee et al. 2000; Muir-Broaddus et al. 2002; Nigg, et al. 1996; Naglieri et al. 2005 y Weiss y Totten, 2004). Esta falta de concordancia ha generado un debate entre los especialistas sobre cuál de las dos estrategias podría ser más pertinente.

Por un lado, algunos autores indican que los profesores poseen un mayor conocimiento sobre los síntomas y el diagnóstico del TDAH (Perlod, Louw y Kleymhans, 2010) y estas supuestas mayores competencias de los profesores, para hacer el diagnostico, se explicarían considerando que los docentes cuentan con mayores oportunidades para observar los comportamientos relacionados con la memoria de trabajo y los déficit cognitivos, en comparación con los padres, por ejemplo (McCandless y O'Laughlin, 2007). Por el contrario, también se ha señalado que la compresión y el conocimiento que poseen los profesores en torno al TDAH tiende a ser escaso, ya que en gran medida no han tenido una capacitación pertinente especialmente en sus aspectos clínicos (Bell et al. 2011).

$\mathrm{Al}$ respecto, en un estudio se señala que el $85 \%$ de los psicólogos informó que para la evaluación del TDAH emplea escalas que son contestadas por profesores (Handler y DuPaul, 2005). Considerando el argumento sobre el mayor conocimiento del niño que tienen los profesores, este dato no deja de ser importante ya que se vuelve un actor relevante en el proceso no solo del diagnóstico sino también del tratamiento contingente destinado a disminuir el impacto negativo en el niño; lo anterior también puede hacerse extensivo a los padres y a la familia en general (Berger, 2011). No obstante, es relevante considerar el potencial sesgo que conlleva la incorporación de los padres profesores y eventualmente profesionales de la salud no especializados en el diagnóstico del TDAH (Berger y Nevo, 2011), ya que efecto de halo o bien la cercanía afectiva con el menor, podría potenciar el aumento o la disminución de su percepción en torno a la psicopatología (Alloway et al. 2009; Gopin, Healey, Castelli, Marks y Halperin, 2010). Considerando lo anterior, aunque el diagnóstico se realice en base a la información de los profesores, se hace recomendable contar adicionalmente con alguna medida de desempeño objetivo.

En relación con las mediciones de rendimiento objetivo, la prueba computarizada de Conners (CPT) coincidió con la totalidad del criterio en la muestra de niños sin TDAH, pero presentó discrepancia en aquellos niños que habían sido diagnosticados positivamente por los profesores, generando 
la duda sobre si los profesores estarían sobre diagnosticando o la prueba computarizada tiende a generar falsos negativos. Una línea de explicación de estas discrepancias podría encontrarse en los criterios de base utilizadas por ambas pruebas. La prueba CAAS-S utiliza criterios DSM-III R, los que se diferencian un tanto de los criterios DSM-IV R que emplea el CPT Conners. Sin embargo, esta diferencia no sería muy relevante ya que existiría un alto grado de superposición entre las dos definiciones diagnósticas del DSM-III R y DSM-IV R; específicamente, Biederman et al. (1997) encontraron un nivel de correspondencia del 93\% entre los sujetos diagnosticados bajo ambos criterios, además de que Biederman et al. (2007) y Biederman, Petty, Evans, Small y Faraone (2010) recientemente utilizaron criterios DSM-III R en sus indagaciones encontrando resultados muy similares a los obtenidos con criterios diferentes.

En otra línea de discusión, algunos autores han referido que este tipo de instrumento computarizado sólo se enfoca a medir una faceta de la atención, lo que podría no ser coincidente con el desempeño del niño en ambientes naturalistas (aula y hogar) (Zelnik, Bennett-Back, Miari, Goez y Fattal-Valevski, 2012). En este sentido, Ogundele, Ayyash y Banerjee (2011) advierten que los CPT no deben ser utilizados aún como la única herramienta diagnóstica del TDAH, se necesita todavía mayor investigación que proporcione evidencia concluyente respecto a su eficacia. Teniendo presente la discordancia entre los resultados de las pruebas, algunos autores han enfatizado el argumento de que el diagnóstico debe ser un proceso integral sustentado en exámenes médicos, historial clínico, antecedentes recopilados mediante entrevistas, aplicación de escalas en padres y profesores y observaciones naturales en distintos ambientes (Cardo et al. 2011; McGough y McCracken, 2000; Solanto y Alvir, 2009;).

Considerando que para el TDAH no existe una prueba clínica terminante (Batstra, Nieweg y Hadders-Algra, 2014) y de manera de otorgar mayor objetividad al proceso, actualmente los sensores inerciales de las pruebas computarizadas se presentan como una herramienta prometedora que puede proporcionar mayor especificidad, al menos para evitar los falsos positivos (O’Mahony, Florentino-Liano, Carballo, Baca-García y Rodríguez, 2014).

Adicionalmente se analizó la posible relación entre el diagnóstico de TDAH y la edad y el sexo de los participantes y al contrario de lo que se podría esperar de acuerdo a los resultados reportados por Urzua, Domic, Cerda, Ramos y Quiroz (2009) no se encontraron diferencias significativas. Urzúa encontró que la prevalencia era mayor en varones entre
6 y 8 años y la muestra de este estudio incluye participantes de 8 años hasta los 16 años, por lo que es probable que la ausencia de relación se explique en razón del rango etario de la muestra.

Siguiendo en la línea de lo señalado en párrafo anterior, se puede considerar una limitación del trabajo la acotada cantidad de participantes, al momento de pretender la generalización de los resultados a otras poblaciones tanto por el rango etario como por el nivel socio económico y la dependencia del colegio de procedencia. El instrumento CAAS-S a su vez, podría requerir probar sus propiedades psicométricas en una muestra más amplia para incrementar los niveles de seguridad sobre la información que proporciona. El trabajo sería el primer estudio que compara el instrumento CAAS-S con una prueba computarizada, además que se une al conjunto de investigaciones que han utilizado el cuestionario en diversas indagaciones (Lambert y Hartsough, 1998; Richards, Symons, Greene y Szuszkiewicz, 1995 y Symons, Greene y Syntons, 1996). Así mismo en la última década se ha reportado su utilización Lambert (2005), Updike (2006), Lambert, McLeod y Schenk (2006), lo que resalta la utilidad del instrumento y de los criterios diagnóstico que emplea.

En conclusión, en este estudio se encontró que los resultados del test computarizado coinciden con el test aplicado a los profesores en descartar la presencia de TDAH en los niños y niñas de la muestra pero solo coincide parcialmente con los participantes del grupo diagnosticado con el trastorno evidenciando un posible sobre diagnóstico cuando se considera la opinión de los profesores o bien enfrentarse al hecho de estar en presencia de mediciones que apuntan a constructos diferentes. Dada la sensibilidad que poseen actualmente los instrumentos existentes, la conjunción y profundización de los mismos en la fase de detección puede resultar importante para el curso del trastorno. Lo anterior podría contribuir a reducir las tasas de sobre diagnóstico que en la actualidad es motivo de debate a nivel global (Batstra et al. 2014; Bruchmüller, Margraf y Schneider, 2012; Bruchmüller y Schneider, 2012). 


\section{Referencias}

Alloway, T. P., Gathercole, S.E., Holmes, J., Place, M., Elliott, J. G., y Hilton, K. (2009). The diagnostic utility of behavioral checklists in identifying children with ADHD and children with working memory deficits. Child Psychiatry y Human Development, 40, 353-366.

American Psychiatric Association. (2000). Diagnostic and Statistical Manual of Mental Disorders Text Revision (DSM-IV-TR), 4th ed. Washington, DC: American Psychiatric Association.

American Psychiatric Association. (2013). Diagnostic and statistical manual of mental disorders (5th ed.). Arlington, VA: American Psychiatric Publishing.

Anderson, D. L., Watt, S. E., Noble, W., y Shanley, D. C. (2012). Knowledge of attention deficit hyperactivity disorder (ADHD) and attitudes toward teaching children with ADHD: The role of teaching experience. Psychology in the Schools, 49, 511-525.

Antshel, K. M., Hargrave, T. M., Simonescu, M., Kaul, P., Hendricks, K. y Faraone, V. (2011). Advances in understanding and treating ADHD. BMC Medicine, 9, 7015-1741.

Barkley, R. A. (1991). The ecological validity of laboratory and analogue assessment methods of ADHD symptoms. Journal of Abnormal Child Psychology, 19, 149-178.

Barkley, R. A., DuPaul, G. J., y McMurray, M. B. (1990). Comprehensive evaluation of attention deficit disorder with and without hyperactivity as defined by research criteria. Journal of Consulting and Clinical Psychology, 58, 775-789.

Batstra, L., Nieweg, E. H., y Hadders-Algra, M. (2014). Exploring five common assumptions on Attention Deficit Hyperactivity Disorder. Acta Paediatrica, 103, 696-700.

Bell, L., Long, S., Garvan, C., y Bussing, R. (2011).The impact of teacher credentials on ADHD stigma perceptions. Psychology in the Schools, 48, 184-197.

Berger, I. (2011). Diagnosis of Attention Deficit Hyperactivity Disorder: Much ado about something. Israeli Medical Association Journal, 13, $571-574$.

Berger, I., y Nevo, Y. (2011). Early developmental cues for diagnosis of attention deficit/hyperactivity disorder in young children. Developmental disabilities research reviews, 17, 170-179.

Berger, I., Slobodin, O., Aboud, M., Melamed, J., y Cassuto, H. (2013) Maturational delay in ADHD: evidence from CPT. Front Human Neuroscience, 7, 1-11.

Biederman, J., Ball, S. W., Mick, E., Monuteaux, M. C., Kaiser, R., Bristol, E., y Faraone, S. (2007). Informativeness of maternal reports on the diagnosis of ADHD: An analysis of mother and youth reports. Journal of Attention Disorders, 10, 410-417

Biederman, J., Faraone, S. V., Weber, W., Russell, R. L., Rater, M., y Park, K. S. (1997). Correspondence between DSM-III-R and DSM-IV atten- tion-deficit/hyperactivity disorder. Journal of the American Academy of Child y Adolescent Psychiatry, 36, 1682-1687.

Biederman, J., Petty, C. R., Evans, M., Small, J., y Faraone, S. V. (2010). How persistent is ADHD? A controlled 10-year follow-up study of boys with ADHD. Psychiatry research, 177, 299-304.

Brown, T. E. (2001). Brown Attention-Deficit Disorder Scales for Children and Adolescents. San Antonio, TX: Psychological Corporation.

Bruchmüller, K., y Schneider, S. (2012). Fehldiagnose Aufmerksamkeitsdefizit-und Hyperaktivitätssyndrom?. Psychotherapeut, 57, 77-89.

Bruchmüller, K., Margraf, J., y Schneider, S. (2012). Is ADHD diagnosed in accord with diagnostic criteria? Overdiagnosis and influence of client gender on diagnosis. Journal of Consulting and Clinical Psychology, $80,128-138$.

Cardo, E., Servera, M., Vidal, C., de Azua, B., Redondo, M., y Riutort, L. (2011). The influence of different diagnostic criteria and the culture on the prevalence of attention deficit hyperactivity disorder. Revista de Neurología, 52, S109-S117.

Cohen, R. (2011). Continuous Performance Tests. In Jeffrey S. Kreutzer, John DeLuca, Bruce Caplan (eds.), Encyclopedia of Clinical Neuropsychology (699 - 701). New York: Springer.

Conners, C. K. (1995). Conners' Continuous Performance Test computer program 3.0 User's manual. Toronto, ON: Multi Health Systems Inc.

Conners, C. K. (2000). Conners' Continuous Performance Test (CPT-II) computer program for windows, technical guide, and software manual. Toronto, ON: Multi Health Systems Inc.

Conners, C. K. (2004). Conner's Continuous Performance Test (CPT-II) V.5. Canadá: Multi Health Systems Inc.

Conners, C.K. (2014). Conners Continuous Performance Test 3rd Edition ${ }^{\mathrm{TM}}$. North Tonawanda: Multi-Health Systems Inc.

Díaz-Orueta, U., Garcia-López, C., Crespo-Eguilaz, N., Sánchez-Carpintero, R., Climent, G., y Narbona, J. (2014). AULA virtual reality test as an attention measure: Convergent validity with Conners. Continuous Performance Test. Child Neuropsychology, 20, 328-342.

DuPaul, G. J., Anastopoulos, A. D., Shelton, T. L., Guevremont, D. C., y Metevia, L. (1992). Multi-method assessment of Attention Deficit Hyperactivity Disorder: The diagnostic utility of clinic-based tests. Journal of Clinical Child Psychology, 21, 394-402.

Edwards, M. C., Gardner, E., Chelonis, J., Schulz, E., Flake, R., y Diaz, P. (2007). Estimates of the Validity and Utility of the Conners' Continuous Performance Test in the Assessment of Inattentive and/or Hyperactive-Impulsive Behaviors in Children. Journal of Abnormal Child Psychology, 35, 393- 404.

Egeland, J., Johansen, S. N., y Ueland, T. (2009). Differentiating between ADHD sub-types on CCPT measures of sustained attention and vigilance. Scandinavian Journal of Psychology, 50, 347-354.

Forbes, G. B. (2001). A comparison of the Conners' Parent and Teacher Rating Scales, the ADD-H Comprehensive Teacher's Rating Scale, 
and the Child Behavior Checklist in the clinical diagnosis of ADHD. Journal of Attention Disorders, 5, 25-40.

Gopin, C., Healey, D., Castelli, K., Marks, D., y Halperin, J. M. (2010). Usefulness of a clinician rating scale in identifying preschool children with ADHD. Journal of Attention Disorders, 13, 479-488.

Gordon, M. (1983). The Gordon Diagnostic System. DeWitt, NY: Gordon Systems.

Greenberg, L. M., y Waldman, I. D. (1993). Developmental normative data on the Test of Variables of Attention (T.O.V.A.). Journal of Child and Adolescent Psychiatry, 34 (6), 1019-1030.

Gupta, R., y Kar, B.K. (2010). Specific Cognitive Deficits in ADHD: A Diagnostic Concern in Differential Diagnosis. Journal of Child and Family Studies, 19, $778-786$.

Halperin, J. M., Wolf, L. E., Pascaulvaca, D. M., Newcorn, J. H., Healey, J. M., O'Brien, J. D., Morganstein, A., y Young, G. (1988). Differential assessment of attention and impulsivity in children. Journal of the American Academy of Child and Adolescent Psychiatry, 27, 326-329.

Handler, M. W., y DuPaul, G. J. (2005). Assessment of ADHD: Differences across psychology specialty areas. Journal of Attention Disorders, 9, 402-412.

Lambert, N. M., Hartsough, C. S. \& Sandoval, J. (1990). The children's attention and adjustment survey. Palo Alto, CA: Consulting Psychologists Press.

Lambert, N. (2005). The contribution of childhood ADHD, conduct problems, and stimulant treatment to adolescent and adult tobacco and psychoactive substance abuse. Ethical Human Psychology and Psychiatry, 7, 197-221.

Homack, S., y Riccio, C.A. (2006). Conners' Continuous Performance Test (2nd ed.;. CCPT-II). Journal Attention Disorder, 9, 556-558.

Kallitsoglou, A. (2013). An investigation of the usefulness of the Conner's Continuous Performance Test II in the assessment of inattention and hyperactivity in the school. European Journal of Special Needs Education, 28, 348-361.

Klee, S. H., y Garfinkel, B. D. (1983). The computerized continuous performance task: a new measure of inattention. Journal of Abnormal Child Psychology, 11, 487-496.

Lambert, N. (2005). The contribution of childhood ADHD, conduct problems, and stimulant treatment to adolescent and adult tobacco and psychoactive substance abuse. Ethical Human Psychology and Psychiatry, 7, 197-221.

Lambert, N. M., y Hartsough, C. S. (1998). Prospective study of tobacco smoking and substance dependencies among samples of ADHD and non-ADHD participants. Journal of Learning Disabilities, 31, 533-544.

Lambert, N. M., Hartsough, C. S., y Sandoval, J. (1990). The children's attention and adjustment survey. Palo Alto, CA: Consulting Psychologists Press.
Lambert, N. M., McLeod, M., y Schenk, S. (2006).Subjective responses to initial experience with cocaine: an exploration of the incentive-sensitization theory of drug abuse. Addiction, 101, 713-725.

Martell, M. M., y Nigg, J. T. (2006). Child ADHD and personality/ temperament traits of reactive and effortful control, resiliency, and emotionality. Journal of Child Psychology and Psychiatry, 47, 1175-1183.

McCandless, S., y O'Laughlin, L. (2007). The clinical utility of the Behavior Rating Inventory of Executive Function (BRIEF) in the diagnosis of ADHD. Journal of Attention Disorders, 10, 381-389.

McGee, R. A., Clark, S. E. y Symons, D. K. (2000). Does the Conners' Continuous Performance Test Aid in ADHD Diagnosis?. Journal of Abnormal Child Psychology, 28, 415-424.

McGough, J. J., y McCracken, J. T. (2000). Assessment of attention deficit hyperactivity disorder: A review of recent literature. Current Opinion in Pediatrics, 12, 319-324.

Miranda, M., Barbosa, T., Muszkat, M., Rodrigues, C., Sinnes, E., Coelho, L., Rizzuti, S., Palma, S., y Bueno, O. (2012). Performance patterns in Conners' CPT among children with attention deficit hyperactivity disorder and dyslexia. Arquivos de Neuro-Psiquiatria, 70, 91-96.

Moldavsky, M., Groenewald, C., Owen, V. \& Sayal, K. (2013). Teachers' recognition of children with ADHD: role of subtype and gender. Child and Adolescent Mental Health, 18 (1), 18-23.

Monastra, V. J. (2008). Unlocking the potential of patients with. ADHD: A model for clinical practice. Washington, DC: American Psychological Association.

Muir-Broaddus, J., Rosenstein, L., Medina, D., y Soderberg, C. (2002). Neuropsychological test performance of children with ADHD relative to test norms and parent behavioral ratings. Archives of Clinical Neuropsychology, 17, 671-689.

Naglieri, J., Goldstein, S., Delauder, B., y Schwebach, A. (2005). Relationships Between the WISC-III and the Cognitive Assessment System with Conners' Rating Scales and Continuous Performance Tests. Archives of Clinical Neuropsychology, 20, 385-401.

Nichols, S. L., y Waschbusch, D. A. (2004). A review of the validity of laboratory cognitive tasks used to assess symptoms of ADHD. Child Psychiatry and Human Development, 34, 297-315.

Nigg, J. T., Hinshaw, S. P., y Halperin, J. M. (1996). Continuous performance test in boys with attention deficit hyperactivity disorder: Methylphenidate dose response and relations with observed behaviors. Journal of Clinical Child Psychology, 25, 330-340.

Ogundele, M. O., Ayyash, H. F., y Banerjee, S. (2011). Role of computerised continuous performance task tests in ADHD. Progress in Neurology and Psychiatry, 15, 8-13.

O’Mahony, N., Florentino-Liano, B., Carballo, J. J., Baca-García, E., y Rodríguez, A. A. (2014). Objective diagnosis of ADHD using IMUs. Medical engineering y physics, 36, 922-926. 
Perlod, M., Louw, C., y Kleymhans, S. (2010). Primary school teachers' knowledge and misperceptions of attention deficit hyperactivity disorder (ADHD). South African Journal of Education, 30, 457-473.

Richards, C. M., Symons, D. K., Greene, C. A., y Szuszkiewicz, T. A. (1995). The bidirectional relationship between achievement and externalizing behavior problems of students with learning disabilities. Journal of learning disabilities, 28, 8-17.

Rizzo, A. A., Bowerly, T., Buckwalter, G., Klimchuk, D., Mitura, R., y Parsons, T. (2006). A virtual reality scenario for all seasons: the virtual classroom. CNS Spectrums, 11 , 35-44.

Sanford, J. A. (1995). Intermediate Visual and Auditory Continuous Performance Test Richmond. VA: Braintrain

Sciutto, M. J., y Eisenberg, M. (2007). Evaluating the evidence for and against the overdiagnosis of ADHD. Journal of Attention Disorders, 11, 106-113.

Shapiro, S. K., y Garfinkel, B. D. (1986). The occurrence of behavior disorders in children: The interdependence of attention deficit disorder and conduct disorder. Journal of the American Academy of Child Psychiatry, 25, 809-819.

Solanto, M. V., y Alvir, J. (2009). Reliability of DSM-IV symptom ratings of ADHD: implications for DSM-V. Journal of Attention Disorder, 13, 107-116

Symons, D., Greene, C., y Syntons, S. (1996). Using Multiple Reporters of Problem Behavior to Predict Clinical Referral of Adolescents with Learning Disabilities. Canadian Journal of School Psychology, 11, 178-190

Tripp, G., Schaughency, E., y Clarke, B. (2006). Parent and teacher rating scales in the evaluation of attention-deficit hyperactivity disorder: contribution to diagnosis and differential diagnosis in clinically referred children. Journal of Developmental y Behavioral Pediatrics, 27, 209-218.

Updike, C. (2006). The use of FM systems for children with attention deficit disorder. Journal of Educational Audiology, 13, 7-14.

Urzúa, A., Domic, M., Cerda, A., Ramos, M., y Quiroz, J. (2009). Trastorno por déficit de atención con hiperactividad en niños escolarizados. Revista chilena de pediatría, 80(4), 332-338.

Weis, R., y Totten, S. J. (2004). Ecological validity of the Conners' Continuous Performance Test - II in a school-based sample. Journal of Psychoeducational Assessment, 22, 47-61.

Winders, D., y Williams, P. G. (2011). Attention deficit/hyperactivity disorder in preschool-age children: issues and concerns. Clinical Pediatrics, 50, 144-152.

Wolraich, M. L., Lambert, W., Doffing, M. A., Bickman, L., Simmons, T., y Worley, K. (2003). Psychometric properties of the Vanderbilt ADHD diagnostic parent rating scale in a referred population. Journal of Pediatric Psychology, 28, 559-568.

Wolraich, M. L., McKeown, R. E., Visser, S. N., Bard, D., Cuffe, S. P., Neas, B., Geryk, L. L., Doffing, M., Bottai, M., Abramowitz, A. J., Beck, L., Holbrook J. R., y Danielson, M. (2014). The Prevalence of ADHD: Its
Diagnosis and Treatment in Four School Districts Across Two States. Journal of Attention Disorders, 18, 563-575.

Zelnik, N., Bennett-Back, O., Miari, W., Goez, H. R., y Fattal-Valevski, A. (2012). Is the test of variables of attention reliable for the diagnosis of attention-deficit hyperactivity disorder (ADHD)? Journal of Child Neurology, 27, 703-707. 